\title{
Design and Realization of Consumption Data Acquisition and Management System
}

\author{
Chuanfei Qiu, Zhichao Shao, Jianhua Tu, Fuqi Qu and Xianglin Tan \\ Ordnance NCO Academy, Arm Engineering University of PLA, Wuhan Hubei 430075, China
}

\begin{abstract}
A consumption data acquisition and management system is developed to take statistics of actual situations and consumption data of maintenance materials and provide basis for defining the maintenance material standards. The system is explained from the aspects of system function design, system structure design and key technologies.
\end{abstract}

Keywords-data acquisition; data management; data synchronization; styling; acquisition terminal

\section{INTRODUCTION}

Maintenance materials as an important material basis for maintaining equipment can be a critical support for efficient after-sales services, and material standards are the basic reference for material guarantee. With the continuous increase of the production and sales volume of products, the supply of material funds is incompatible with the demand for materials, and there are gaps between material guarantee and equipment research and development, between material guarantee efficiency and precise equipment warranty. The material standards are compiled on the basis of material consumption data and material indicators of reliability, maintainability and economy. The material consumption data, which reflect the actual demand for equipment and materials to a certain extent, are the primary basis for the standard formulation. Therefore, a consumption data acquisition and management system is developed to collect statistics of actual situations and consumption data of maintenance materials, form the actual consumption regularity and provide basis for defining material standards. This system is explained herein from the aspects of system function design, system structure design and key technologies.

\section{SYSTEM FUNCTION DESIGN}

The consumption data acquisition and management system comprises two core subsystems: handheld acquisition terminal and consumption data management software. It can achieve the acquisition of $1 \mathrm{D}$ and 2D barcode data, local data and database summary and management, as well as centralized processing of equipment information. The system has the functions of consumption data acquisition, data management, data query and display, data analysis and data synchronization.

\section{A. Consumption Data Acquisition Function}

The consumption data acquisition function means that the handheld acquisition terminal can acquire 1D and 2D barcode data, store them in local database for management, enable centralized processing of acquired information, or even upload to the database of the data management for synchronous management.

\section{B. Consumption Data Management Function}

The consumption data management mainly enables acquisition and input of material barcode information, manual entry of information, record of material stock-in/out information, statistics of material stock and consumption data, classified management of stock and material consumption data, bulk import and export of data; and management of data management software accounts to enhance the data confidentiality and security.

\section{Consumption Data Query and Display Function}

The data query and display function can inquire and display material stock and consumption data, related electronic texts, and circuit diagrams, which can be zoomed in or out.

\section{Consumption Data Analysis Function}

The consumption data analysis function can display the material consumption data in the data form of graphics and charts, including generation of statistical graphs for total records, stock change, marked quantity change, stock-in/out records and marked records. The system can support the extension of other data statistical analysis function and call of third-party tools. The system also has the abilities of extending big data analysis, calculation and statistics and provides analysis results in the form of graphics or texts.

\section{E. Consumption Data Synchronization Function}

The consumption data synchronization function can support regular data backup, complete data transmission, update and synchronization between data management software and handheld acquisition terminal, export data in the system and support the network reporting function.

\section{SYSTEM STRUCTURE DESIGN}

\section{A. System Architecture}

The consumption data acquisition and management system needs to achieve the centralized management of maintenance material consumption information, and to improve the maintenance guarantee efficiency in terms of maintenance and management. The system has the functions of barcode input, query, printing, bulk import, group management and 
consumption trend display.

The system consists of two parts: software subsystem and hardware subsystem. The software subsystem is the consumption data management software, which is PC software and requires support of SQL Server database. The hardware subsystem includes handheld acquisition terminals, barcode scanner, and barcode printer. Its composition is shown in Figure I.

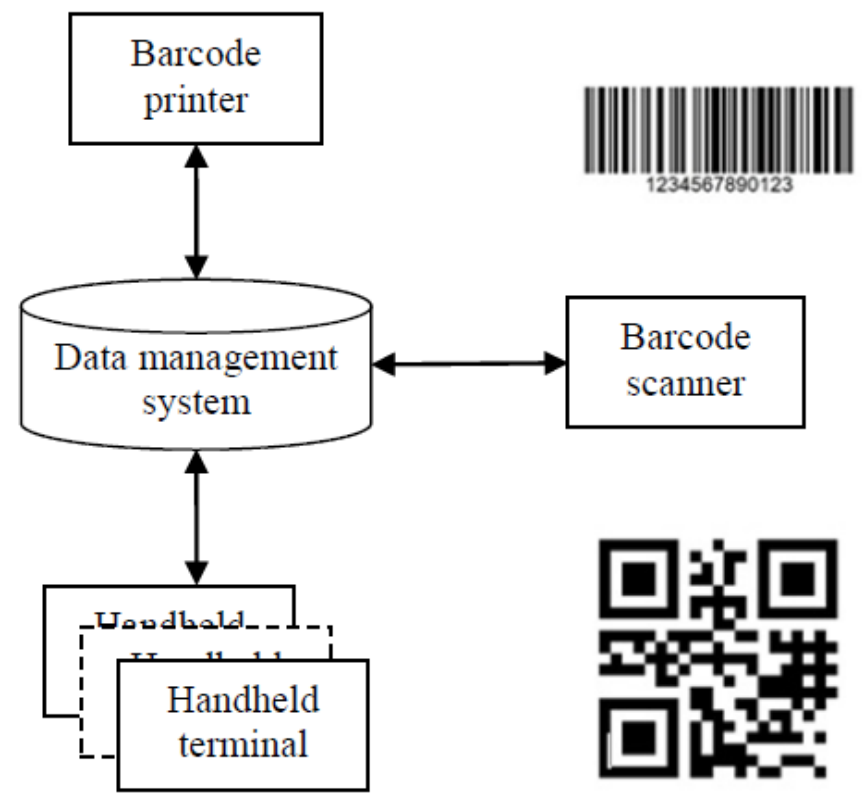

FIGURE I. ARCHITECTURE OF CONSUMPTION DATA ACQUISITION AND MANAGEMENT SYSTEM

The consumption data management software supports Windows XP, Windows 7 x86 32-bit and Windows 7 x64-bit operating systems.

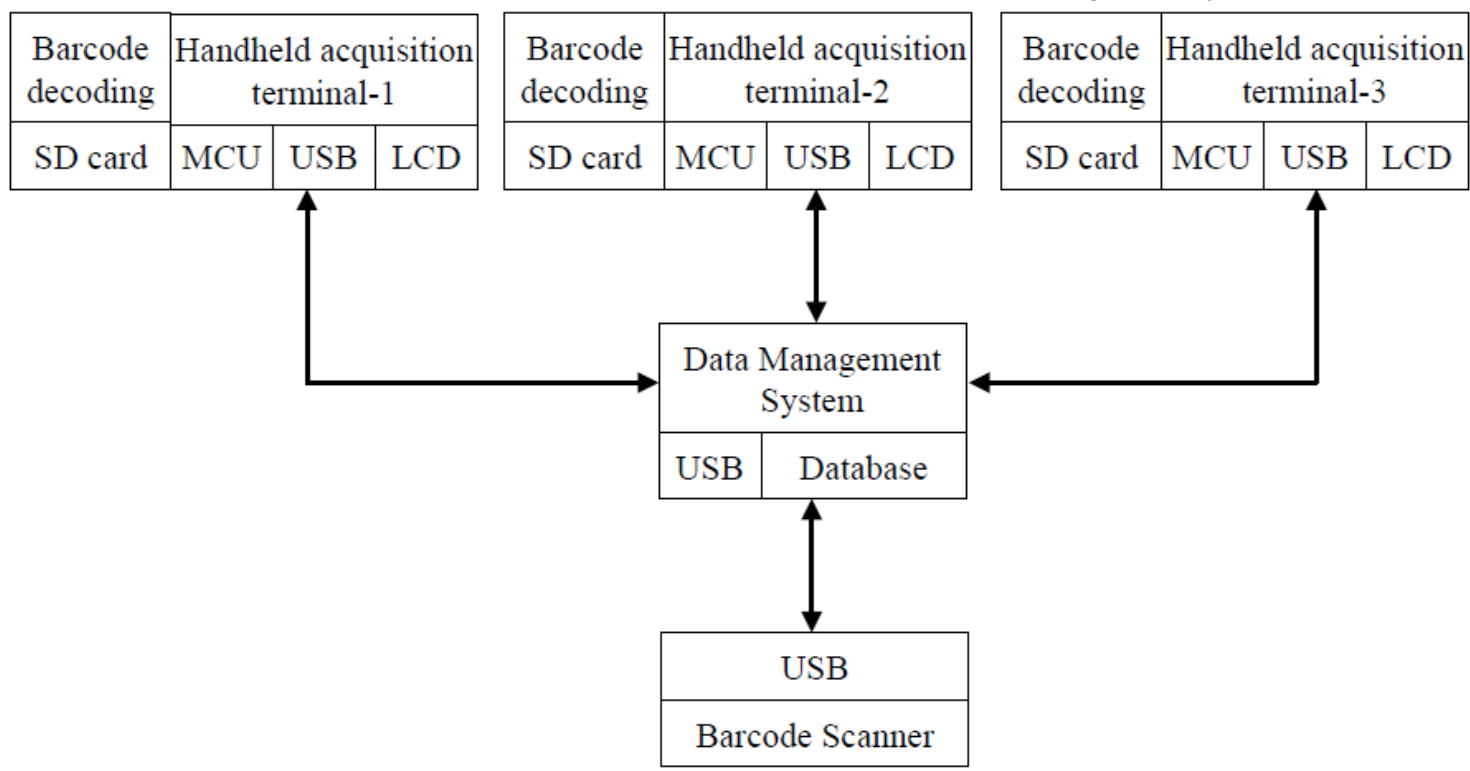

In order to facilitate practical use, the handheld acquisition terminals adopt a key-plus-touch operation mode, which enables entry of basic information such as letters and numbers, supports the acquisition and storage of field pictures (photos), supports the review and modification of database data contents, and supports query of relevant senior information of equipment, such as schematics and circuit diagrams.

\section{B. System Composition}

The overall design block diagram of the consumption data acquisition and management system is shown in Figure II.

The consumption data acquisition and management system adopts a single-master/ multi-slave design framework, and multiple handheld collection terminals are used as operation terminals to realize the identification and entry of data information such as 1D and 2D barcodes, and store them in the local database. The host of the consumption data management software runs as a host to achieve the data summary, recording and analysis of all handheld acquisition terminals. Meanwhile, the host is equipped with a barcode scanner, which is used for direct scanning and entry of barcode information.

The consumption data management software is mainly composed of three parts: USB port driver module, humancomputer interface, and database management. The handheld acquisition terminals include five parts: barcode identification module, USB port driver module, touch LCD operation module, local database management module, and SD card management. These terminals can work independently. The host and acquisition terminals use the SD card to synchronize local database with server database. For USB connection, the handheld acquisition terminals become data acquisition stations for synchronizing data between host and handheld acquisition terminals, and achieving the data synchronization management. The barcode scanner as a component of hot can realize identification and upload of 1D and 2D barcodes and synergize with the host data management system. 


\section{Data Management Design}

The handheld acquisition terminals have local database designed with basic operations such as query, addition and modification. Given the process capability and actual need for use of the core system, the streamlined data structure management is adopted. The SD card is used for the storage of automatic backup data, and it can support a maximum of 32G SD card. The core advantage of choosing an SD card as the data storage carrier is pluggable so that it is easy for the synchronization of computer management software and equipment database and the management of data files, and ensures that data files are not lost even if the equipment does not work properly.

The basic management operations of the database are realized by the management software, which must take account of the consistency and compatibility with the database content of the computer management software, so as to facilitate synchronous management. The formats of data storage need to be specially designed for the hardware environment of the equipment. The data management diagram is shown in Figure III.

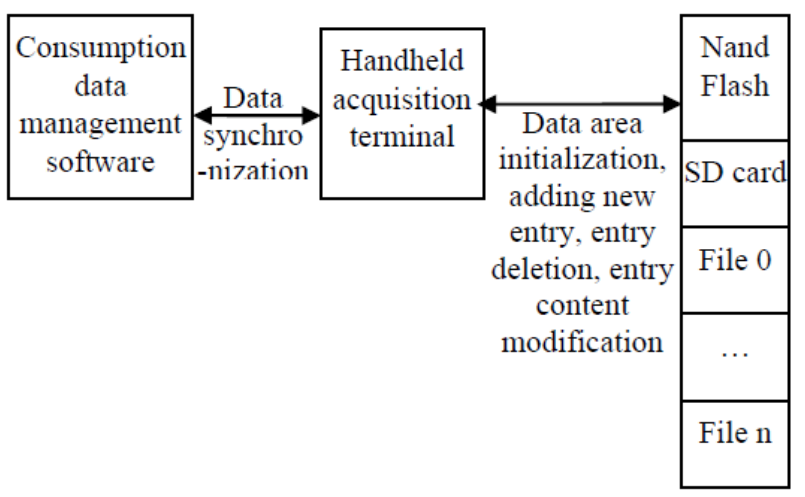

FIGURE III. DATABASE MANAGEMENT DESIGN

The automatic data backup adopts the user trigger mode. When the user completes the related operations of the data acquisition device and manually quits the application software, the program automatically backs up latest key files to the SD card backup directory, such as product entries and operation records, thereby ensuring the data reliability.

If the handheld acquisition terminals are unable to acquire data normally due to accidental data destruction or system crash, the SD card can be used to obtain historical key data and enhance the reliability of data storage.

\section{KEY TECHNOLOGIES}

\section{A. Handheld Acquisition Terminal Development}

The core of the hardware part of handheld acquisition terminals is the $1 \mathrm{D}$ and $2 \mathrm{D}$ barcode identification module. At present, there are many types of relevant modules on the market with similar performance. The CMOS cameras are used to identify 2D barcodes and compatible with 1D barcode reading. The working principles are similar and these products are mature. The CMOS camera module selected for this system has 500W pixels, use ARM-Cortex A8 as the control core, and support autofocus, with the scanning frequency of $100 \pm 20$ times/second, the recognition angle of 5-35 degrees, high recognition rate and quick recognition speed [1]. It can reach the performance index of current mainstream products, and be fully applicable to the application scenario of the system. The hardware module purchases products from a leading manufacturer in China, which has been specialized in the barcode recognition field for more than 10 years. The hardware is stable and reliable and adopts the state-of-art technology. This product has been widely used in warehouse management, supermarkets, express delivery industry, outdoor inspections, etc., and the hardware module is made of advanced technology with stable and reliable performance.

The software part of handheld acquisition terminals uses Wince6.0 as the system platform, which is a widely applied mainstream embedded operating system on the market. It is suitable for secondary development because the source code is open source. Wince 6.0 is an operating system based on Windows operating system and developed specifically for the embedded environment [2]. 6.0 is a new system version that is mostly used in consumer electronic products and industrial control environments. ARM-Cortex A8 core can match well with both. The system platform built based on the platform has a high degree of compatibility with hardware resources and is suitable for secondary development of the application layer software.

\section{B. Data Storage Management}

SQL Server is a relational database management system introduced by Microsoft Corporation. It is easy to use and has good scalability. It is highly integrated with LabView and can be used across multiple platforms from Microsoft Windows 98 to Microsoft Windows 2012 [3].

The consumption data management software development platform selects LabView graphical development environment as the development software. LabView is a graphical development software developed by National Instruments Corporation. It is different from the language development software such as $\mathrm{C}$ and $\mathrm{C}++$, and its graphical development environment is conducive to building test platforms and industrial control platforms quickly and professionally. LabView is widely used in the fields of industrial testing and industrial control. It is well-known for its strong professionalism and high ease of use, and is suitable for crossplatform development. LabView 8.6 is the most used and most stable version of all LabView distributions, so it is selected as the consumption data management software development environment [4]. It can achieve all functional indicators with high development efficiency, professional interface and perfect support for cross-platform migration.

The data management adopts a distributed architecture design based on the PC SQL Server database, and builds relatively independent local database on handheld acquisition terminals. Synchronization of data files is achieved through online synchronization so as to systematically realize the distributed architecture and apply to actual management environments of military equipment and material consumption. 


\section{CONCLUSIONS}

Through the designs of functions, architecture and key technologies of the consumption data acquisition and management system, the handheld acquisition terminals are studied, the consumption data management software is developed, and the distributed acquisition and management, query and display, analysis and synchronization of material consumption data can be comprehensively realized. Statistics of actual situations and consumption data of equipment and material guarantee are collected for analyzing actual consumption rules and provide basis for the formulation of material standards. Also, the consumption data acquisition and management system can be applied to other models of equipment or other types of consumption data acquisition and management for solving the problems of other equipment.

\section{REFERENCES}

[1] Wang Heng, In-depth analysis of ARM Cortex-A8. Biejing: Electronics Industry Press, 2016, pp. 297-298.

[2] Feng Zhangdongsong, Chen Fangguo, Windows CE 6 embedded advanced programming. Biejing: Tsinghua University Press, 2009, pp. 34-36.

[3] MingRi Tech, SQL Server from the beginning to the master. Biejing: Tsinghua University Press, 2017, pp. 25-27.

[4] Song Ming, LabVIEW programming detailed solution. Biejing: Electronics Industry Press, 2017, pp.10-12 